\title{
Overexpression of Rcan1-1L Inhibits Hypoxia-Induced Cell Apoptosis through Induction of Mitophagy
}

\author{
Lijun Sun*, Yuewen Hao, Rui An, Haixun Li, Cong Xi, and Guohong Shen
}

\begin{abstract}
Mitophagy, a cellular process that selectively targets dysfunctional mitochondria for degradation, is currently a hot topic in research into the pathogenesis and treatment of many human diseases. Considering that hypoxia causes mitochondrial dysfunction, which results in cell death, we speculated that selective activation of mitophagy might promote cell survival under hypoxic conditions. In the present study, we introduced the Regulator of calcineurin 1-1L (Rcan1-1L) to initiate the mitophagy pathway and aimed to evaluate the effect of Rcan1-1L-induced mitophagy on cell survival under hypoxic conditions. Recombinant adenovirus vectors carrying Rcan1-1L were transfected into human umbilical vein endothelial cells and human adult cardiac myocytes. Using the 3-(4,5Dimethylthiazol-2-yl)-2,5-diphenyltetrazolium bromide MTT assay and Trypan blue exclusion assay, Rcan1-1L overexpression was found to markedly reverse cell growth inhibition induced by hypoxia. Additionally, Rcan1-1L overexpression inhibited cell apoptosis under hypoxic conditions, as detected by annexin V-fluorescein isothiocyanate (FITC)/propidium iodide (PI) apoptosis assay. Meanwhile, the mitochondria-mediated cell apoptotic pathway was inhibited by Rcan1-1L. In contrast, knockdown of Rcan1$1 \mathrm{~L}$ accelerated hypoxia-induced cell apoptosis. Moreover, Rcan1-1L overexpression significantly reduced mitochondrial mass, decreased depolarized mitochondria, and downregulated ATP and reactive oxygen species production. We further delineated that the loss of mitochondrial mass was due to the activation of mitophagy induced by Rcan1-1L. Rcan1-1L overexpression activated autophagy flux and promoted translocation of the specific mitophagy receptor Parkin into mitochondria from the cytosol, whereas inhibition of autophagy flux resulted in the accumulation of Parkin-loaded mitochondria. Finally, we demonstrated that mitochondrial permeability transition
\end{abstract}

Department of Radiology, Xijing Hospital, The Fourth Military Medical University, Xi'an, Shaanxi 710032, P.R. China

*Correspondence: lijunsunxian@126.com

Received 25 April, 2014; revised 10 September, 2014; accepted 11 September, 2014; published online 5 November, 2014

Keywords: apoptosis, hypoxia, mitochondria, mitophagy, Rcan1-1L pore opening was significantly increased by Rcan1-1L overexpression, which suggested that Rcan1-1L might evoke mitophagy through regulating mitochondrial permeability transition pores. Taken together, we provide evidence that Rcan1-1L overexpression induces mitophagy, which in turn contributes to cell survival under hypoxic conditions, revealing for the first time that Rcan1-1Linduced mitophagy may be used for cardioprotection.

\section{INTRODUCTION}

Cellular protein and organelle quality are under tight control in order to maintain cellular homeostasis (Goldberg, 2003). Besides the ubiquitin-proteasome system, a process named autophagy has been found that eliminates and catabolizes the cytosol, aggregated proteins, and dysfunctional organelles (Nakatogawa et al., 2009; Yang and Klionsky, 2010). It is intriguing that organelles can be specifically targeted for degradation through autophagy. Currently, there is great interest in mitophagy, a process that can selectively recognize and remove dysfunctional mitochondria (Ding and Yin, 2012; Song et al., 2014; Youle and Narendra, 2011). Understanding the role of mitophagy may provide novel insights for the development of therapeutic treatments for many human diseases.

Mitochondria are organelles critical for the life of the cell, acting as its main energy converters and ATP supply for cellular processes (Campello et al., 2014). However, dysfunctional mitochondria can be detrimental for the cell due to the release of reactive oxygen species (ROS) and the activation of apoptotic pathways (Scherz-Shouval and Elazar, 2011). Under hypoxic conditions, mitochondria are vulnerable to damage, especially in cardiomyocytes, which often suffer ischemic injury (Chatterjee et al., 2002; Nakagawa et al., 2005; Neubauer, 2007). Mitochondrial dysfunction has been related to a wide range of diseases including cancer and various neurological diseases (Cho et al., 2010; Koopman et al., 2012; Nunnari and Suomalainen, 2012). Therefore, efficient removal of dysfunctional mitochondria is beneficial for cell protection under stress stimuli. Currently, one strategy for specific removal of mitochondria has been characterized, named mitophagy, which was first reported by Lemasters (2005). In mammalian cells, several mitophagy pathways have been reported including the Parkin/PTENinduced kinase protein 1 (PINK1)-mediated mitophagy pathway 
and the Nix/Bnip3-mediated mitophagy pathway (Youle and Narendra, 2011). Parkin, with E3 ubiquitin ligase activity, can be quickly recruited to the mitochondria by PINK1, which accumulates upon mitochondrial membrane depolarization to ubiquitylate mitochondrial proteins that activate mitophagy (Youle and Narendra, 2011). Nix and Bnip3 have been reported to bind to microtubule-associated protein light chain 3 (LC3) for autophagolysosome membrane formation during autophagy and mediate the entry of the mitochondrion into an autophagosome (Novak et al., 2010; Zhang and Ney, 2009). Interestingly, Nix has recently been found to promote Parkin translocation to mitochondria from the cytosol (Chan et al., 2011). More recently, a mitochondrial protein, Fundc1, has been characterized as a new mediator of mitophagy that interacts with LC3 for autophagolysosome membrane formation during autophagy and regulates entry of the mitochondrion into an autophagosome for degradation (Liu et al., 2012). Recent studies have made considerable advances in mitophagy and have helped facilitate understanding the precise mechanism of mitophagy. However, more investigations are needed to gain a better understanding of the role of mitophagy in the pathogenesis of diseases and the underlying mechanism.

Regulators of calcineurin (Rcan) have drawn particular interest recently, as they play an important role in cellular processes and pathogenesis (Serrano-Candelas et al., 2014). To date, three Rcan genes have been characterized, including Rcan1, Rcan2, and Rcan3 (Davies et al., 2007), of which Rcan1, a stress-induced protein, has been widely studied in recent years (Crawford et al., 1997; Davies et al, 2007; Ermak et al., 2011; Wang et al., 2002). To date, three isoforms of Rcan1 have been characterized, including Rcan1-1L, Rcan1-1S, and Rcan1-4 (Davies et al, 2007; Ermak et al., 2002; 2006). It has been demonstrated that Rcan1 has a protective effect on cells under stress stimuli such as calcium overload (Ermak et al, 2002; Zhao et al., 2008). In Drosophila, Rcan1 has been reported to interact with mitochondrial adenine nucleotide translocators, implying a function of Rcan1 in mitochondria (Chang and Min, 2005). Recently, a study has found that Rcan $1-1 \mathrm{~L}$ specifically induces mitophagy and efficient mitochondrial degradation (Ermak et al., 2012), which implies that Rcan1-1L may be useful as a novel target for inducing mitophagy in order to treat diseases.

Clinical cardiac surgery usually causes ischemia in cardiomyocytes, leading to myocardial damage and pathological remodeling. Ischemia- and hypoxia-induced mitochondrial dysfunction are the main reasons for cardiomyocyte damage (Chatterjee et al., 2002; Nakagawa et al., 2005; Neubauer, 2007). Currently, overcoming ischemia- and hypoxia-induced damage to cardiomyocytes is a challenge for the treatment of ischemia-related heart disease. In the present study, we aimed to investigate whether Rcan1-1L overexpression would activate mitophagy and whether the effect would be beneficial for cell survival under hypoxic conditions.

\section{MATERIALS AND METHODS}

\section{Cell culture}

Human umbilical vein endothelial cells (HUVECs) were purchased from the American Type Culture Collection (ATCC, USA) and cultured in Dulbecco's modified eagle medium (DMEM; Invitrogen, USA) supplemented with $10 \%$ fetal bovine serum (FBS) and $1 \%$ penicillin/streptomycin. Human adult cardiac myocytes (HACMs) were obtained from ScienCell (ScienCell, USA) and maintained in cardiac myocyte medium (ScienCell) supplemented with $5 \%$ FBS and $1 \%$ penicillin/streptomycin. All cells were cultured in an incubator (Life Technologies, USA) under a humidified atmosphere with $5 \% \mathrm{CO}_{2}$ at $37^{\circ} \mathrm{C}$.

Recombinant adenovirus construction and transfection Recombinant adenovirus vectors were supplied by Biowit Technologies (Biowit, China). Briefly, the cDNA encoding Rcan1-1L was amplified and subcloned into the adenoviral shuttle vector pAd-CMV; green fluorescent protein (GFP) was used as a non-specific control. The adenoviral shuttle vector pAd-CMV and adenoviral gene expression vector pAdEasy-1 were then homologously recombined in Escherichia coli strain BJ5183. The obtained recombinant plasmids were than transfected into 293 cells to produce recombinant adenovirus. The recombinant adenoviruses were harvested and the titers were determined using the p24 ELISA kit (Cell Biolabs, USA) before use. Cells were administered recombinant adenovirus vectors and cultured under normoxic conditions or subjected to hypoxia for $12,24,36$, or $48 \mathrm{~h}$. To induce hypoxia, cells were paced in a hypoxia chamber containing $95 \% \mathrm{~N}_{2}$ and $5 \% \quad \mathrm{CO}_{2}$ at $37^{\circ} \mathrm{C}$. Cells treated with phosphate-buffered saline (PBS) were used as a control.

\section{Small interference RNA (siRNA) transfection}

Cell transfection of siRNA was performed according to the manufacture's instructions. A total of $1 \mu \mathrm{g}$ Rcan $1-1 \mathrm{~L}$ siRNA or non-specific siRNA generated by Genepharma (China) were diluted in $500 \mu \mathrm{l}$ cardiac myocyte medium containing $5 \mu \mathrm{l}$ lipofectamine (Invitrogen, USA) and incubated for $30 \mathrm{~min}$ at room temperature. Then, the mixtures $(100 \mu \mathrm{l}$ per well) were added to the cells and further cultured for $24 \mathrm{~h}$ under hypoxic conditions. Thereafter, the cells were harvested for further analysis.

\section{Western blot analysis}

Proteins were extracted from cells using cell lysis buffer and the protein concentration was quantified by the Bradford assay. A total of $20 \mu \mathrm{g}$ of protein were separated by $12 \%$ sodium dodecyl sulfate polyacrylamide gel electrophoresis (SDS-PAGE) and then transferred to a nitrocellulose membrane (Amersham, UK), which was incubated with $2 \%$ nonfat dry milk in Trisbuffered saline (TBS) to block non-specific binding at room temperature (RT) for $1 \mathrm{~h}$. After blocking, the membrane was incubated in blocking buffer containing primary antibodies overnight at $4^{\circ} \mathrm{C}$. Anti-Rcan1-1L antibody was purchased from Sigma (USA), while other antibodies used in the present study, including anti-cytochrome c, anti-AIF, anti-Bcl-2, anti-TOM20, anti-calnexin, anti-GM130, and anti-Parkin, were purchased from Santa Cruz Biotechnology (USA). Subsequently, the membrane was washed with TBS-Tween (TBST) for $10 \mathrm{~min}$ and incubated with horseradish peroxidase (HRP)-conjugated secondary antibody (Boster Corporation, China) diluted in blocking buffer for $1 \mathrm{~h}$ at room temperature. After being washed again with TBST buffer for $10 \mathrm{~min}$, proteins were detected using enhanced chemiluminescence (Pierce, USA).

\section{Cell growth and viability assay}

Cell growth and viability were assessed by the 3-(4,5Dimethylthiazol-2-yl)-2,5-diphenyltetrazolium bromide (MTT) assay according to a standard method. Briefly, cells were seeded in 96-well plates and cultured until $80 \%$ confluence was reached. After the cells had been administered recombinant adenovirus vectors $\left[1 \times 10^{6}\right.$ plaque-forming units $(\mathrm{pfu})$ of virus per well], they were then cultured under normoxic conditions or subjected to hypoxia for $6,12,24$ or $48 \mathrm{~h}$. Thereafter, the culture medium was replaced by fresh medium and $20 \mu \mathrm{l}$ PBS 
containing $5 \mathrm{mg} / \mathrm{ml}$ MTT (Sangon, China) were added per well. The cells were incubated for an additional $4 \mathrm{~h}$. A total of $150 \mu \mathrm{l}$ dimethyl sulfoxide were added per well for $15 \mathrm{~min}$ to dissolve the formazan crystals. Finally, the absorbance was measured at $490 \mathrm{~nm}$ using an ELISA reader (Bio-tek Instruments, USA). The experiment was performed in quadruplicate and was repeated thrice.

\section{Trypan blue exclusion assay}

The dead cells were measured by using the Trypan blue exclusion assay. Cells infected with adenovirus vectors $\left(1 \times 10^{7} \mathrm{pfu}\right)$ or transfected with siRNA $(0.002 \mu \mathrm{g} / \mu \mathrm{l})$ in 6 -well plate were cultured under normoxic conditions or hypoxic conditions for 24 $\mathrm{h}$. Then the cells were harvested and trypsinized followed by centrifugation $(1000 \times g$ for $5 \mathrm{~min})$. The pellet was collected and washed with PBS and then re-suspended in PBS. An equal volume of $0.4 \%$ trypan blue solution was added into the cell suspension and incubated for $5 \mathrm{~min}$. Both the dead cells and living cells were observed and counted using a Cellometer (Nexcelom Bioscience, USA).

\section{Fluorescence-activated cell sorting (FACS) analysis}

The rate of cell apoptosis was assessed using the annexin $\mathrm{V}$ fluorescein isothiocyanate (FITC)/propidium iodide (PI) apoptosis detection kit (Cusabio, China) according to the supplier's instructions. Briefly, the cells were transfected with recombinant adenovirus vectors or siRNA cells in 6-well plate under normoxic conditions or hypoxic conditions for $24 \mathrm{~h}$. Then, cells were harvested by centrifugation (1000 $\mathrm{g}$ for $5 \mathrm{~min}$ ), washed with ice-cold PBS and resuspended in $195 \mu \mathrm{l}$ of binding buffer. Thereafter, the cells were incubated with annexin $\mathrm{V}$ stock solution $(5 \mu \mathrm{l})$ at $4^{\circ} \mathrm{C}$ for 30 $\mathrm{min}$ in the dark. The cells were centrifuged (1000 $\mathrm{g}$ for $5 \mathrm{~min}$ ) and resuspended in $195 \mu \mathrm{l}$ of binding buffer. Then, a total of $5 \mu$ of $\mathrm{PI}$ were added and the cells were incubated for $5 \mathrm{~min}$ at room temperature in the dark. Finally, the cells were analyzed by a FACS analyzer (BD Biosciences, USA).

For the analysis of mitochondrial mass, the cells were incubated with 200 nM Mitotracker Green FM (Invitrogen, USA) in PBS for 20 min at $37^{\circ} \mathrm{C}$ after transfected with recombinant adenovirus vectors $\left(1 \times 10^{7} \mathrm{pfu}\right)$ in 6 -well plate for $24 \mathrm{~h}$ under hypoxia. After washing with PBS for three times, the fluorescence was detected using a FACS flow cytometer. Mitochondrial mass was evaluated by comparing the mean fluorescence signals between cells transfected with recombinant adenovirus vectors and those transfected with PBS.

MPTP opening was determined as previously described (Petronilli et al., 1999). Briefly, the infected cells $\left(1 \times 10^{7} \mathrm{pfu}\right)$ were cultured under hypoxic conditions for 24,48 or $72 \mathrm{~h}$ in 6well plate. Then, the cells were treated with $1 \mu \mathrm{M}$ calcein-AM (Molecular Probes) at $37^{\circ} \mathrm{C}$ in the dark for $30 \mathrm{~min}$. Afterward, 1 $\mathrm{mM} \mathrm{CoCl} 2$ (Sigma, USA) was added and the cells were incubated for another $10 \mathrm{~min}$ in the dark at $37^{\circ} \mathrm{C}$. Thereafter, the fluorescence signal of mitochondria-trapped calcein was analyzed using a FACS flow cytometer at $530 \mathrm{~nm}$ excitation wavelength. For each experiment, calcein fluorescence from 30,000 cells was acquired and median value analysis was conducted using FlowJo software.

\section{ROS measurement}

Cells seeded on 24-well plates were infected with recombinant adenovirus vectors $\left(6 \times 10^{6} \mathrm{pfu}\right)$ and cultured under hypoxic conditions for $24 \mathrm{~h}$. After the cells were collected and washed with PBS, they were incubated with $50 \mu \mathrm{M}$ 2',7'dichlorodihydrofluorescein diacetate (DCFH-DA; Sigma, USA) at $37^{\circ} \mathrm{C}$ for $45 \mathrm{~min}$ in the dark. After washing thrice with PBS, the fluorescence was quantified using a Multi-Detection microplate reader (Bio-tek Instruments, USA) with an excitation wavelength of $485 \mathrm{~nm}$ and emission wavelength of $530 \mathrm{~nm}$. The experiment was performed in quadruplicate and was repeated thrice.

Measurement of ATP levels

The cellular ATP level was measured using an ATP assay kit (Beyotime, China) following the manufacturer's instructions. Briefly, cells infected with recombinant adenovirus vectors $\left(1 \times 10^{7} \mathrm{pfu}\right)$ in 6-well plate for $24 \mathrm{~h}$ under hypoxia were harvested and homogenized in $200 \mu \mathrm{l}$ of somatic cellular ATP releasing reagent, incubated on ice for $15 \mathrm{~min}$, and centrifuged at $14,000 \times g$ for 15 $\min$ at $4^{\circ} \mathrm{C}$. The supernatant was collected and added into the detecting tube containing $100 \mu$ of ATP detection solution, and the bioluminescence were subsequently measured using a Synergy HT luminescence plate reader (Biotek, USA).

Measurement of mitochondrial membrane potential Mitochondrial membrane potential was assessed by fluorescent JC-1 dye according to a previously reported method (Cossarizza et al., 1996). In polarized mitochondria, JC-1 accumulates in the mitochondrial matrix and forms red fluorescent $\mathrm{J}$-aggregates, while, in depolarized mitochondria, JC-1 exists as green monomers. Briefly, the treated cells were harvested, washed with PBS and incubated with the JC-1 fluoroprobe (2.5 $\mu \mathrm{g} / \mathrm{ml}$ ) for $30 \mathrm{~min}$ at $37^{\circ} \mathrm{C}$ in the dark. Cells were harvested $(1000 \times g$ for $5 \mathrm{~min})$ and washed twice with $1 \times$ incubation buffer. The cells were resuspended in $500 \mu$ of $1 \times$ incubation buffer and fluorescent signals were determined by a FACS flow cytometer.

Mitochondrial and cytosol protein extraction

Mitochondrial and cytosolic fractions were isolated using an EnoGene $^{\mathrm{TM}}$ Mitochondria Protein Extraction Kit (EnoGene, China) according to the manufacturer's instructions. Briefly, the cells were harvested and homogenized in ice-cold lysis buffer I containing $1 \mu \mathrm{l}$ protease inhibitor, $10 \mu \mathrm{l}$ phosphatase inhibitor, and $5 \mu \mathrm{l}$ phenylmethanesulfonyl fluoride $(100 \mathrm{mM})$ followed by centrifugation at $800 \mathrm{~g}$ for $5 \mathrm{~min}$ at $4^{\circ} \mathrm{C}$. Then, the supernatant was collected and added into $500 \mu \mathrm{l}$ mitochondria isolation reagent $A$, followed by centrifugation at $15,000 \times g$ for $20 \mathrm{~min}$ at $4^{\circ} \mathrm{C}$. The supernatant containing the cytosolic fraction was collected and the pellet, enriched in mitochondria, was rinsed with rinsing buffer followed by centrifugation at $15,000 \times g$ for $10 \mathrm{~min}$ at $4^{\circ} \mathrm{C}$. The supernatant was discarded and the pellet was resuspended in ice-cold lysis buffer II containing $1 \mu$ l protease inhibitor, $10 \mu \mathrm{l}$ phosphatase inhibitor, and $5 \mu \mathrm{l}$ phenylmethanesulfonyl fluoride $(100 \mathrm{mM})$, followed by centrifugation at $12,000 \mathrm{rpm}$ for 15 $\min$ at $4^{\circ} \mathrm{C}$. The supernatant containing mitochondrial proteins was then collected for further analysis.

\section{Statistical analysis}

Data are presented as mean \pm standard deviation (SD). Comparisons between two groups and among multiple groups were conducted by Student $t$-test and one-way analysis of variance (ANOVA), respectively. A p value of less than 0.05 was considered statistically significant. All statistical analyses were performed using SPSS version 11.5 (SPSS Inc., USA).

\section{RESULTS}

Overexpression of Rcan1-1L impairs cell growth and viability under normoxic conditions

To explore the potential effect of Rcan1-1L on cell growth, we first analyzed the effect of overexpression of Rcan1-1L on cell 
A

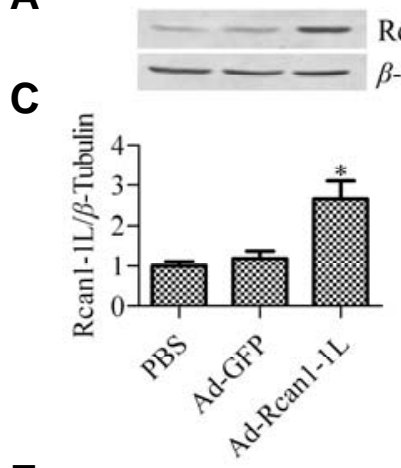

E

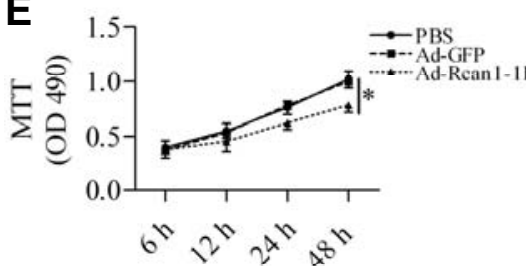

G

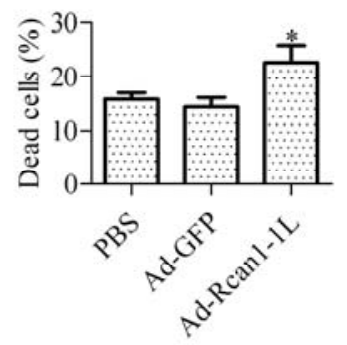

Rcan1-1L $\beta$-Tubulin
B

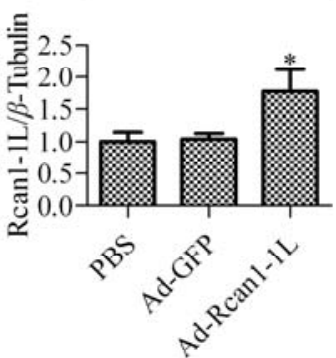

$\mathbf{F}$

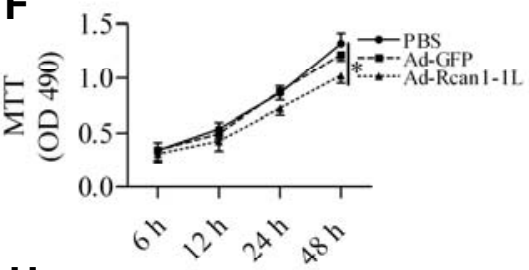

H

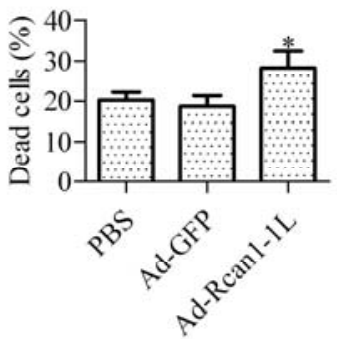

Rcan1-1L $\beta$-Tubulin
Fig. 1. Effect of Rcan1-1L overexpression on cell growth and viability under normoxic conditions. Western blot analysis of levels of Rcan1$1 \mathrm{~L}$ protein in HACMs (A) and HUVECs (B) with different treatments. PBS, cells treated with PBS; Ad-GFP, cells infected with null vectors; Ad-Rcan1-1L, cells infected with recombinant adenovirus vectors expressing Rcan1-1L. Cells infected with recombinant adenovirus vectors were cultured for $24 \mathrm{~h}$ under normoxic conditions $\left(5 \% \mathrm{CO}_{2}\right.$ at $\left.37^{\circ} \mathrm{C}\right)$ before the cells were collected for analysis. Cells treated with PBS were taken as control. Relative levels of Rcan1$1 \mathrm{~L}$ protein in HACMs $(C)$ and HUVECs (D) with different treatments were analyzed using Image-Pro Plus 6.0 software and normalized to $\beta$ Tubulin. The MTT assay was performed to assess cell growth and viability in HACMs (E) and HUVECs $(F)$ with the treatments indicated above. The infected cells were cultured for 6 , 12,24 , and $48 \mathrm{~h}$ under normoxic conditions before harvested for MTT analysis. Cell death in HACMs $(\mathrm{G})$ and HUVECs $(\mathrm{H})$ was measured using the Trypan blue exclusion assay. Cells with the treatments indicated above were cultured for $24 \mathrm{~h}$ under normoxic conditions. Then the cells were harvested, trypsinized and stained with $0.4 \%$ trypan blue solution for 5 $\min$. Both the dead cells and living cells were observed and counted using a Cellometer. ${ }^{*} P<$ 0.05 vs Ad-GPF or PBS group. growth and viability under normoxic conditions. Recombinant adenovirus vectors carrying Rcan1-1L (Ad-Rcan1-1L) or GFP (Ad-GFP) were used to infect HACMs and HUVECs. After incubating for $24 \mathrm{~h}$, expression of Rcan1-1L protein was measured by Western blot analysis. The results showed that AdRcan1-1L infection significantly increased Rcan1-1L levels by 2.65-fold and 1.77-fold in HACMs (Figs. 1A and 1C) and HUVECs (Figs. 1B and 1D), respectively, compared with control cells transfected with PBS. We then analyzed the effect of Rcan1-1L overexpression on cell growth and viability by the MTT assay. The results showed that Rcan1-1L overexpression suppressed the cell growth and viability of HACMs (Fig. 1E) and HUVECs (Fig. 1F) under normoxic conditions. Moreover, Trypan blue exclusion assay showed that cell death was significantly greater in Rcan1-1L transfected HACMs (Fig. 1G) and HUVECs (Fig. 1H) than control groups under normoxic conditions.

Overexpression of Rcan1-1L reduces hypoxia-induced inhibition of cell growth and viability

To further verify the effect of Rcan1-1L on cell growth, we then analyzed the effect of Rcan1-1L overexpression on cell growth and viability under hypoxic conditions. The results showed that Rcan1-1L protein expression was slightly increased under hypoxic conditions in Ad-GFP- and PBS-treated groups compared with untreated cells cultured under normoxic conditions (Figs. $2 \mathrm{~A}$ and $2 \mathrm{~B}$ ), implying that Rcan1-1L was a stress-induced protein (Ermak et al., 2002; Zhao et al., 2008). The MTT assay showed that cell growth and viability were significantly inhibited in Ad-GFP- and PBS-treated cells under hypoxic conditions compared with untreated cells under normoxic conditions.
However, in Ad-Rcan1-1L-transfected cells, overexpression of Rcan1-1L significantly reversed the cell growth arrest induced by hypoxia compared with Ad-GFP- and PBS-treated cells under hypoxic conditions (Fig. 2C). In addition, Trypan blue exclusion assay showed that cell death was significantly greater in Ad-GFP- and PBS-treated cells under hypoxic conditions compared with untreated cells under normoxic conditions, whereas overexpression of Rcan1-1L significantly inhibited cell death induced by hypoxia compared with Ad-GFP- and PBStreated cells under hypoxic conditions (Fig. 2D).

Overexpression of Rcan1-1L inhibits hypoxia-induced mitochondria-mediated cell apoptosis

Hypoxic stress generally causes cell death through induction of mitochondria-mediated cell apoptosis, especially in cardiomyocytes, which are particularly vulnerable under hypoxia (Chatterjee et al., 2002). Here, we used HACMs to further delineate the function of Rcan1-1L in hypoxia-induced cell apoptosis. Compared with untreated cells under normoxic conditions, an increase in apoptotic cells was observed in cells treated with PBS or Ad-GFP under hypoxic conditions. In contrast, cells transfected with AdRcan1-1L showed a significant reduction of apoptotic cells under hypoxic conditions compared with cells treated with PBS or Ad-GFP (Fig. 3A). To further determine the effect of Rcan1-1L overexpression on the mitochondria-mediated apoptotic pathway, we determined the levels of cytochrome $c$ and AIF, which are predominantly found in mitochondria under normal conditions. We found that hypoxia activated the apoptotic pathway, leading to leakage of cytochrome $\mathrm{c}$ and AIF into the cytosol from mitochondria. However, in Rcan1-1L-overexpressing cells, this leak- 


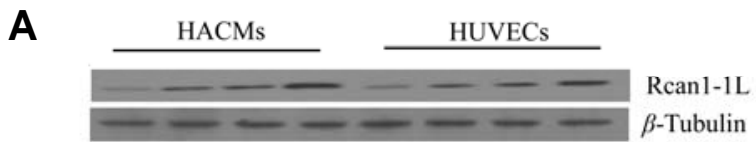

B

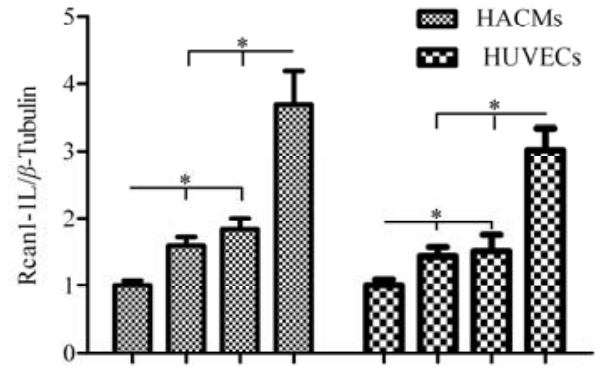

C
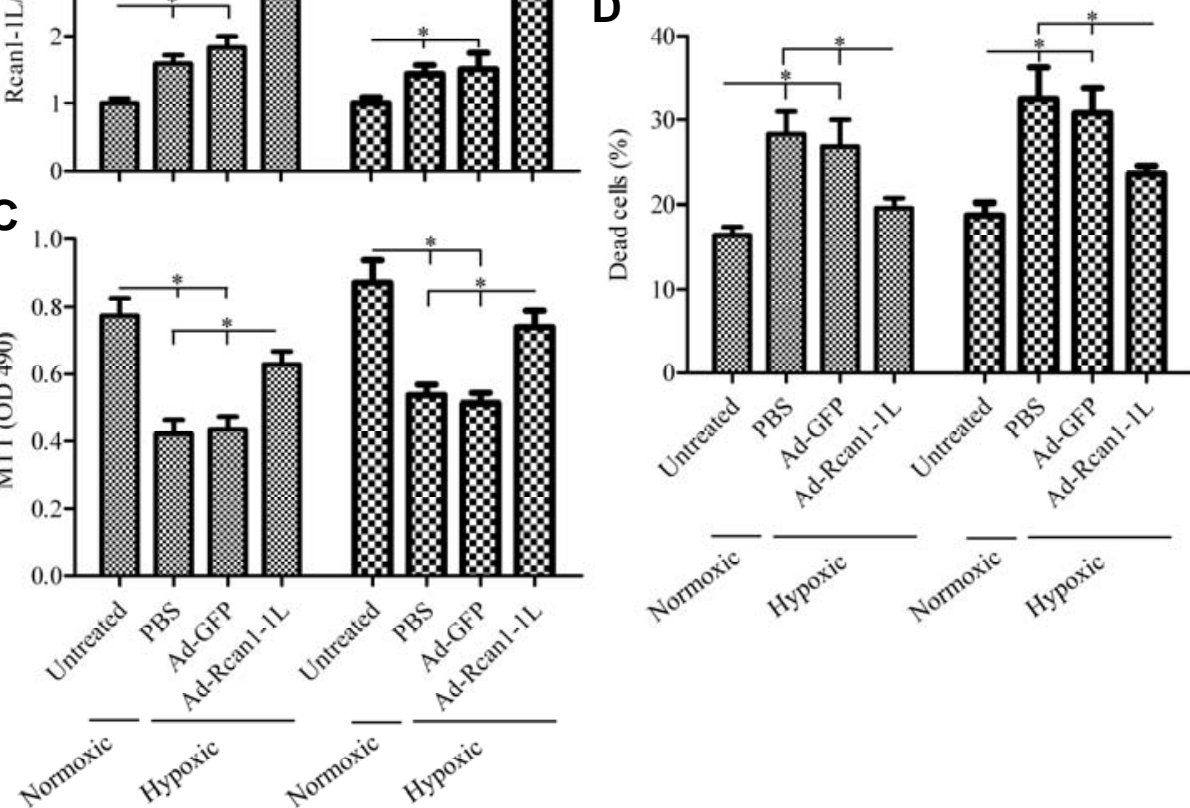

Fig. 2. Effect of Rcan1-1L overexpression on cell growth and viability of HACMs and HUVECs under hypoxic conditions. (A) Western blot analysis was performed to determine the levels of Rcan1-1L in different treated groups of HACMs and HUVECs. Untreated, cells without any treatment were cultured under normoxic conditions $\left(5 \% \mathrm{CO}_{2}\right.$ at $\left.37^{\circ} \mathrm{C}\right)$. PBS, cells treated with PBS; Ad-GFP, cells infected with null vectors; Ad-Rcan1-1L, cells infected with recombinant adenovirus vectors expressing Rcan1-1L; these three cell groups were cultured under hypoxic conditions $\left(95 \% \mathrm{~N}_{2}\right.$ and $5 \% \mathrm{CO}_{2}$ at $\left.37^{\circ} \mathrm{C}\right)$. The different treated cell groups were cultured for 24 before the cells were harvested for analysis. (B) Relative quantification of Rcan1-1L protein expression levels in the different cell groups were analyzed using Image-Pro Plus 6.0 software and normalized to $\beta$-Tubulin. (C) The MTT assay was performed to determine cell growth and viability. Different cell groups treated as above were cultured under the indicated conditions for $24 \mathrm{~h}$ before the cells were harvested for MTT assay. (D) Cell death was measured using Trypan blue exclusion assay. Different cell groups treated as above were cultured under the indicated conditions for $24 \mathrm{~h}$ before the cells were harvested for trypan blue staining. The cells were stained with $0.4 \%$ trypan blue solution and counted using a Cellometer. Data are expressed as mean $\pm \mathrm{SD},{ }^{*} P<0.05$ vs. PBS or Ad-GPF group.

age of cytochrome $\mathrm{c}$ and AIF into the cytosol was suppressed (Fig. 3B). In addition, we also monitored Bcl-2, an anti-apoptotic protein, and activated caspase-3, an apoptosis executioner caspase. The results showed that overexpression of Rcan1-1L increased levels of $\mathrm{Bcl}-2$ protein and inhibited the levels of cleaved caspase- 3 compared to cells transfected with PBS and Ad-GFP under hypoxic conditions (Fig. 3C).

To further confirm the effect of Rcan1-1L on hypoxia-induced cell apoptosis, we silenced gene expression of Rcan $1-1 \mathrm{~L}$ by siRNA in HACMs and detected the effect of Rcan1-1L knockdown on hypoxia-induced cell apoptosis. The results showed that knockdown of Rcan1-IL (Fig. 4A) aggravated hypoxiainduced cell apoptosis as compared with a non-specific siRNA transfected control group (Fig. 4B). Moreover, leakage of cytochrome $\mathrm{C}$ and AIF into the cytosol from mitochondria was increased by Rcan1-1L knockdown (Fig. 4C). Additionally, Rcan $1-1 \mathrm{~L}$ silencing augmented the protein levels of cleaved caspase-3 and reduced the protein levels of Bcl-2 (Fig. 4D). Overall, these results suggested that Rcan1-1L protected cardiomyocytes against hypoxia-induced cell apoptosis.

Overexpression of Rcan1-1L reduces mitochondrial mass To further determine the effect of Rcan $1-1 \mathrm{~L}$ overexpression on mitochondria, we evaluated alterations in mitochondrial mass upon Rcan1-1L overexpression. After transfection and incubation of cells for $24 \mathrm{~h}$, the mitochondria were labeled with MitoTracker Green and their fluorescence intensity was measured. The data showed that overexpression of Rcan1-1L caused a marked decrease in mitochondrial mass compared with cells treated with PBS and Ad-GFP (Fig. 5A). To further confirm the loss of mitochondria, we detected the levels of TOM20 protein, a mitochondrial marker, which was significantly decreased upon Rcan1-1L overexpression, whereas levels of the endoplasmic reticulum marker calnexin and the Golgi body marker GM130 
A

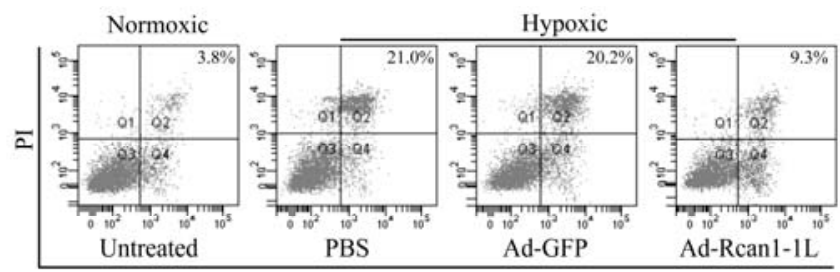

AnnexinV
B

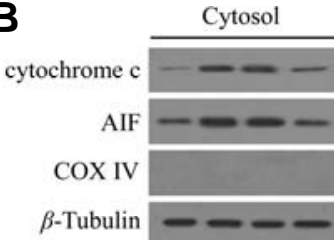

\section{Mitochondria}

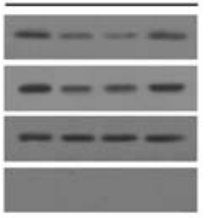

C

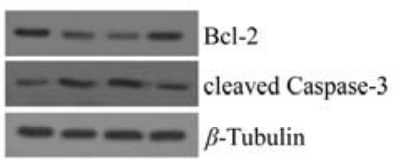

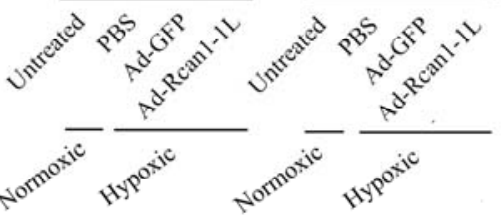

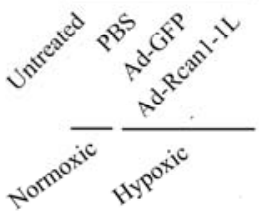

Fig. 3. Effect of Rcan1-1L overexpression on hypoxia-induced cell apoptosis. (A) Cell apoptotic rate was detected by flow cytometry using an annexinV-PI staining method. Untreated, cells without any treatment were cultured under normoxic conditions. PBS, cells treated with PBS; Ad-GFP, cells treated with null vectors; Ad-Rcan1-1L, cells were infected with recombinant adenovirus vectors expressing Rcan1-1L; these three groups were cultured under hypoxic conditions. After cells were cultured for $24 \mathrm{~h}$ under indicated conditions, they were harvested and stained by annexin $\mathrm{V}$ and PI according to manufacture's instruction. Apoptotic cells were detected by FACS flow cytometer. (B) Cytosol and mitochondrial fractions was analyzed by Western blotting with antibodies against cytochrome $c$ and AIF in different treated cell groups as above. The $\beta$-Tubulin was used as the internal control for cytosol proteins. COX IV was used as the internal control for mitochondria proteins. (C) Western blot analysis of Bcl-2 and cleaved caspase-3 in total cell proteins from different treated groups described as above. The $\beta$ Tubulin was used as internal control.
A

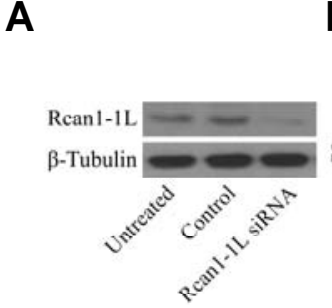

B

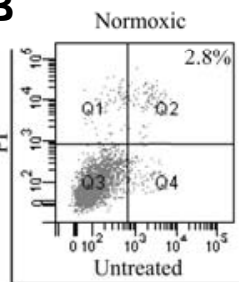

C
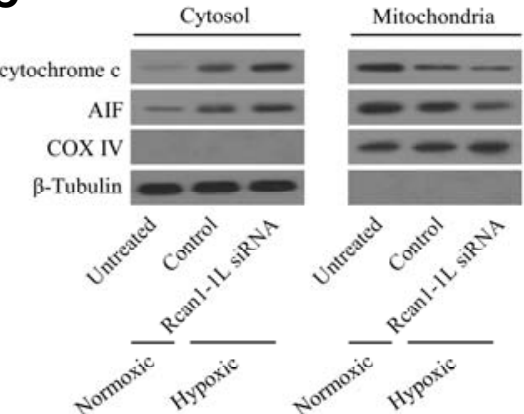

Hypoxic

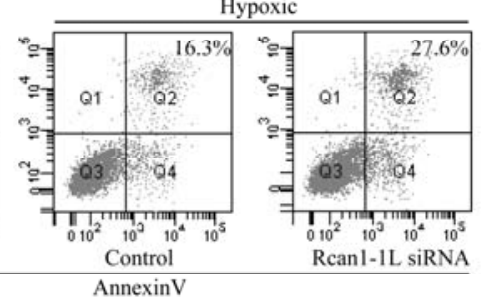

D

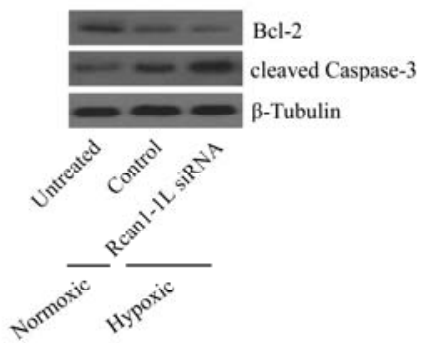

Fig. 4. Effect of Rcan1-1L overexpression on hypoxia-induced cell apoptosis. (A) Western blot analysis to detect the expression of Rcan1$1 \mathrm{~L}$ in different treated cell groups. Untreated, cells cultured under normoxic conditions without treatment; Control, cells transfected with non-specific siRNA and cultured under hypoxic conditions; Rcan1-1L siRNA, cells transfected with Rcan1-1L specific siRNA and cultured under hypoxic conditions. The cells were cultured under normoxic or hypoxic conditions as indicated as above for $24 \mathrm{~h}$ before the cells were harvested for analysis. (B) Cell apoptotic rate was detected by flow cytometry using an annexinV-PI staining method in the different treatment groups. After cells were cultured for $24 \mathrm{~h}$ under indicated conditions as above, they were harvested and stained by annexin $\mathrm{V}$ and $\mathrm{PI}$ according to manufacture's instruction. Apoptotic cells were detected by FACS flow cytometer. (C) Cytosol and mitochondrial fractions in different treated groups were analyzed

by Western blotting with antibodies against cytochrome $\mathrm{c}$ and AIF. The $\beta$-Tubulin was used as the internal control for cytosol proteins. COX IV was used as the internal control for mitochondrial proteins. (D) Western blot analysis of Bcl-2 and cleaved caspase-3 in total cell proteins from different treated groups described as above. The $\beta$-Tubulin was used as internal control.

were basically unchanged (Figs. $5 \mathrm{~B}$ and $5 \mathrm{C}$ ). To further confirm whether the damaged mitochondria were removed, the extent of depolarization of the mitochondria was measured and the results demonstrated that Rcan $1-1 \mathrm{~L}$ overexpression significantly prevented hypoxia-induced mitochondrial depolarization (Fig. 5D). Moreover, damaged mitochondria generate excessive ROS; therefore, we then determined whether Rcan1-1Lmediated mitochondrial degradation could decrease the levels of ROS. As shown in Fig. 5E, overexpression of Rcan1-1L sig- nificantly reduced the intracellular levels of ROS induced by damaged mitochondria under hypoxic conditions. In addition, Rcan1-1L overexpression markedly decreased cellular ATP levels as compared with control groups (Fig. 5F). The data suggested that Rcan1-1L overexpression accelerated mitochondrial degradation and inhibited ROS and ATP production.

Overexpression of Rcan1-1L stimulates mitophagy A recent study demonstrated that Rcan $1-1 \mathrm{~L}$ specifically causes 
A
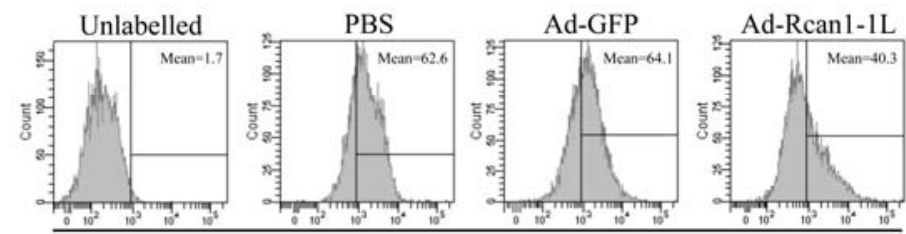

Fluorescence intensity

B

C
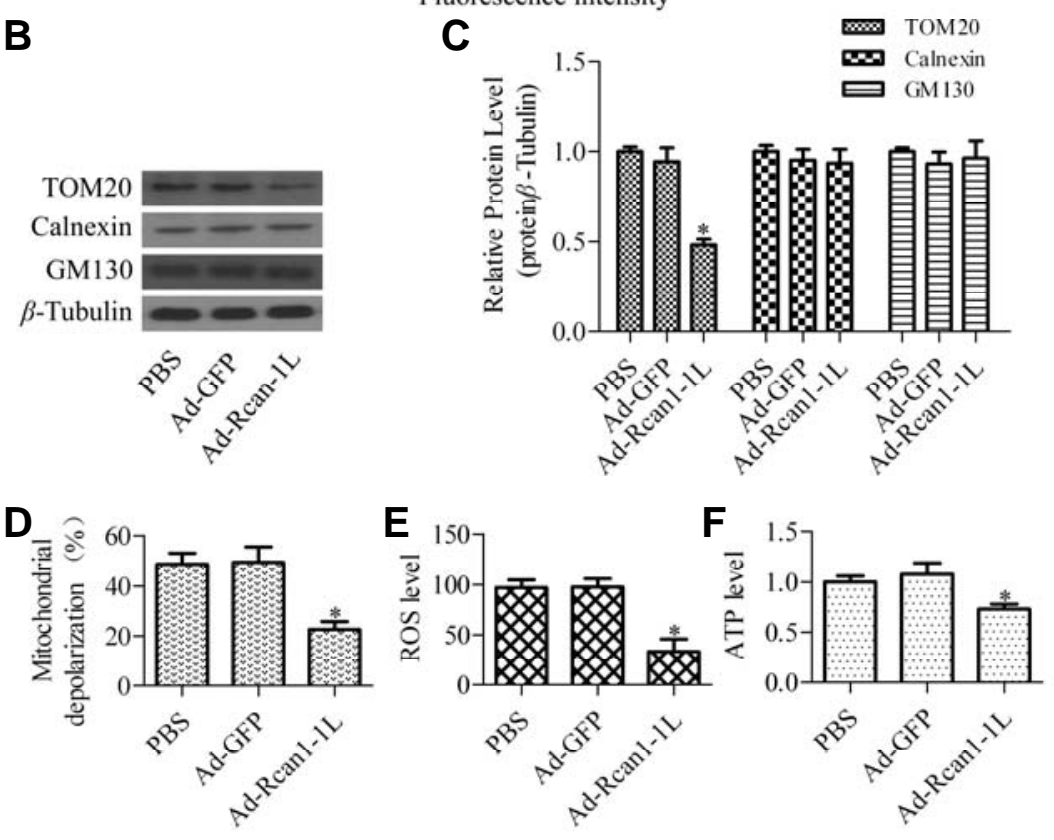

Fig. 5. Effect of Rcan1-1L overexpression on mitochondrial mass. (A) Mitochondrial mass was measured by FACS in different treated cell groups. PBS, cells treated with PBS; Ad-GFP, cells treated with null vectors; Ad-Rcan1-1L, cells were infected with recombinant adenovirus vectors expressing Rcan $1-1 \mathrm{~L}$. The different treated cell groups were cultured under hypoxic conditions for $24 \mathrm{~h}$ and then, incubated with $200 \mathrm{nM}$ Mitotracker Green for $20 \mathrm{~min}$ to label the mitochondria. After washing with PBS, the fluorescence in different cell groups was measured by a FACS flow cytometer. (B) Western blot analysis was performed to detect the levels of TOM20, calnexin, and GM130 with the indicated antibodies in the different treated cell groups. Cells treated with PBS, Ad-GFP, or AdRcan1-1L for $24 \mathrm{~h}$ under hypoxic conditions were harvested for analysis. (C) Relative protein expression levels of TOM20, calnexin, and GM130 in the different cell groups were analyzed using Image-Pro Plus 6.0 software and normalized to $\beta$-Tubulin. (D) Mitochondrial depolarization was assessed using $\mathrm{JC}-1$ dye staining method. Different treated groups as indicated as above were harvested and incubated with JC-1 dye $(2.5 \mu \mathrm{g} / \mathrm{ml})$ for $30 \mathrm{~min}$ at $37^{\circ} \mathrm{C}$ in the dark. After washing with incubation buffer, cells were resuspended in incubation buffer and fluorescent signals were determined by a FACS flow cytometer. (E) ROS levels were determined using the DCFH-DA assay. Different treated groups as indicated as above were harvested and incubated with $50 \mu \mathrm{M} \mathrm{DCFH-DA}$ at $37^{\circ} \mathrm{C}$ for 45 min in the dark. After washing thrice with PBS, the fluorescence was quantified using a Multi-Detection microplate reader. (F) ATP levels were measured by luciferase assay. Different treated groups as indicated as above were harvested and homogenized in $200 \mu$ of somatic cellular ATP releasing reagent. After incubation for $15 \mathrm{~min}$ on ice, cells were harvested by centrifugation $\left(14,000 \times g\right.$ for $15 \mathrm{~min}$ at $\left.4^{\circ} \mathrm{C}\right)$. The ATP levels in the supernatant were detected by ATP detection solution, and the bioluminescence were subsequently measured using a Synergy HT luminescence plate reader. Data are expressed as mean $\pm \mathrm{SD},{ }^{*} P<0.05$ vs Ad-GPF or PBS group.

mitophagy, which accelerates the degradation of mitochondria (Ermak et al., 2012). We speculated that the protection of cells from hypoxia-induced apoptosis might be due to Rcan1-1Lmediated mitophagy that promoted the removal of mitochondria damaged by hypoxia. Hence, we analyzed the effect of Rcan1$1 \mathrm{~L}$ overexpression on autophagy flux. We found that markers of autophagy, LC3 II and autophagy related gene (ATG) 5 (Klionsky et al., 2012), were highly upregulated by Rcan1-1L overexpression, which was further increased by the lysosome inhibitor chloroquine (Klionsky et al., 2012) (Fig. 6A). These data implied that Rcan1-1L evoked autophagy flux. To further verify that the autophagy flux was mitophagy-specific, we then analyzed the mitophagy receptor Parkin, which is mainly found in the cytosol under basal conditions (Matsuda et al., 2010). The results showed that Parkin had translocated into mitochondria from the cytosol upon Rcan1-1L overexpression, which was further increased by chloroquine treatment, implying that inhibition of autophagy flux resulted in the accumulation of the Parkin-loaded mitochondria (Fig. 6B). In summary, Rcan1-1L evoked the mitophagy pathway to degrade mitochondria.

Overexpression of Rcan1-1L leads to mitochondrial permeability transition pore (MPTP) opening

To further explore the underlying mechanism of Rcan1-1L in inducing mitophagy, we determined the effect of Rcan1-1L on mPTP, which plays an important role in regulating mitophagy (Rodriguez-Enriquez et al., 2004; 2009). The results showed that mPTP significantly increased post-Rcan1-1L challenge at 24,48 and $72 \mathrm{~h}$ as compared with $0 \mathrm{~h}$. Also, in comparison with null vector infected groups, mPTP was remarkably increased by Rcan 1-1L overexpression at 24,48 and $72 \mathrm{~h}$, whereas the null vector had no apparent effect on mPTP (Fig. 7). These results suggested that Rcan $1-1 \mathrm{~L}$ increased mitophagy through regulating $\mathrm{MPTP}$.

\section{DISCUSSION}

In the current study, we provide evidence that Rcan1-1L overexpression activates mitophagy, which is in line with the findings of Ermak et al. (2012). We found that overexpression of Rcan1-1L impaired cell growth and viability under normal conditions due to loss of mitochondria. However, under hypoxic conditions, overexpression of Rcan $1-1 \mathrm{~L}$ reduced hypoxia-induced cell growth arrest and apoptosis. The underlying mechanism might be Rcan1-1L-activated mitophagy, which accelerated the clearance of dysfunctional mitochondria attributed to excessive ROS production and activation of the apoptotic pathway.

The human Rcan1 gene (also named Adapt78, calcipressin1, 
A

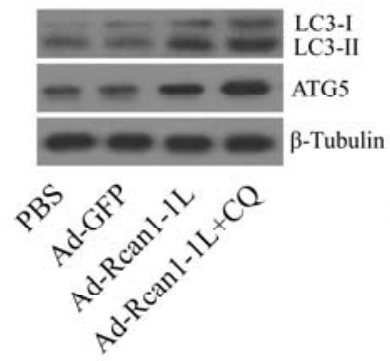

B

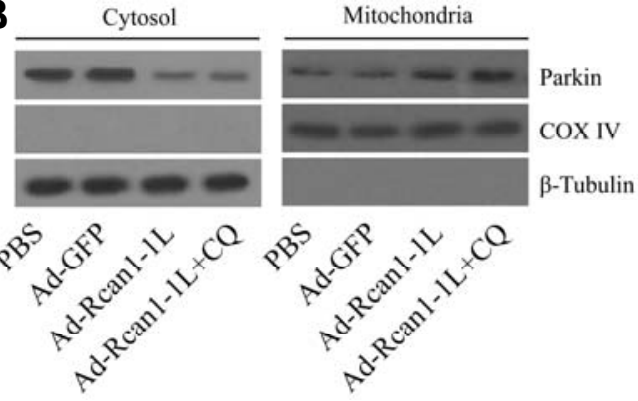

Fig. 6. Effect of Rcan1-1L overexpression on mitophagy. (A) Western blot to detect the protein levels of LC3 and ATG5 in different treated cell groups with the indicated antibodies. PBS, cells treated with PBS; Ad-GFP, cells treated with null vectors; Ad-Rcan1-1L, cells were infected with recombinant adenovirus vectors expressing Rcan1-1L without chloroquine (CQ) treatment; Ad-Rcan1-1L+CQ, cells were infected with recombinant adenovirus vectors expressing Rcan1-1L with chloroquine $(10 \mu \mathrm{M})$ treatment. Different treated cells were cultured under hypoxic conditions for $24 \mathrm{~h}$ and harvested for analysis. (B) Western blot analysis to detect the subcellular location of Parkin in the cytosolic and mitochondrial fractions in different treated cell groups as described above. The $\beta$-Tubulin was used as the internal co ntrol for cytosol proteins. COX IV was used as the internal control for mitochondrial proteins.

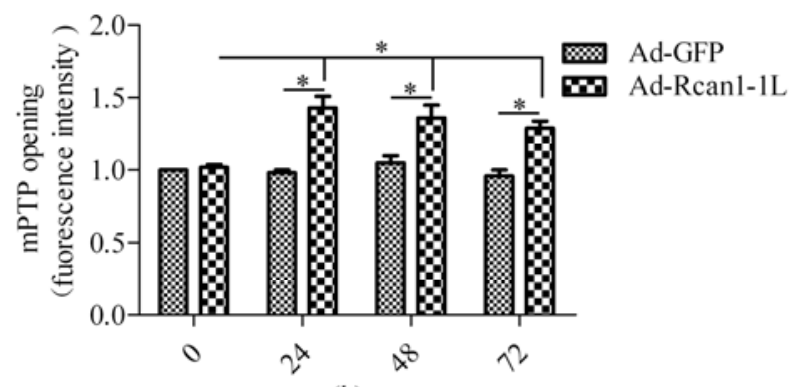

(h)

Fig. 7. Effect of Rcan1-1L overexpression on mPTP. Cells infected with recombinant adenovirus vectors including Ad-GFP or AdRcan1-1L were cultured under hypoxic conditions for 24,48 or $72 \mathrm{~h}$. Afterwards, cells were treated with $1 \mu \mathrm{M}$ calcein-AM and $1 \mathrm{mM}$ $\mathrm{CoCl}_{2}$ at $37^{\circ} \mathrm{C}$ in the dark. The fluorescence signal of mitochondriatrapped calcein was analyzed using a FACS flow cytometer. Mean fluorescence values were converted to arbitrary units. Fluorescence values in null vector-infected cells were set at 1.0. Data are expressed as mean $\pm \mathrm{SD}$, which represent measurements from three independent experiments. ${ }^{*} P<0.05$ vs. Ad-GFP.

Down syndrome candidate region-1, or modulatory calcineurininteracting protein 1) is located on chromosome 21 (Fuentes et al., 1995). The major isoforms of Rcan1 are Rcan1-1 and Rcan1-4, which are encoded by exon 1 and exon 4, respectively (Fuentes et al., 1997). Because the coding sequence of Rcan1-1 contains two AUG initiation sites, it can express two proteins: Rcan1-1L, with 255 aa (the long form) and Rcan1-1S, with 197 aa, (the short form) (Wu and Song, 2013). Rcan1-1L and Rcan1-1S show a wide tissue distribution including the heart, skeletal muscles, and nervous system (Fuentes et al., 1995; 1997). Different isoforms of Rcan1-1 may have distinct functions. Rcan1-1S has been reported to induce cell apoptosis (Sun et al., 2011). However, Rcan1-1L has been demonstrated to promote the proliferation of endothelial cells (Qin et al., 2006). Also, Rcan1-1L was found to protect cells from oxidative stress, but long-term expression of Rcan1-1L facilitated apoptosis $(\mathrm{Wu}$ and Song, 2013). A recent study indicated that Rcan1-1 suppressed hydrogen peroxide-induced cell death through the cAMP response element-binding protein (CREB) and its target gene Bcl-2 (Kim et al., 2013). Consistent with these findings, we demonstrated that Rcan1-1L overexpression inhibited apoptosis induced by hypoxia. We found that Rcan1-1L overexpression significantly inhibited activation of the mitochondria- mediated apoptotic pathway. We also found that ROS levels were downregulated by Rcan $1-1 \mathrm{~L}$ overexpression. These results imply that the cell survival stress induced by damaged mitochondria is eased by Rcan1-1L under hypoxia.

The effective clearance of dysfunctional mitochondria contributes to cell survival under oxidative stress. Thus, we speculated that Rcan1-1L activated mitochondrial degradation. As expected, we found that Rcan $1-1 \mathrm{~L}$ overexpression significantly decreased mitochondrial mass, while other organelles remained unchanged. Further data indicated that Rcan1-1L activated the mitophagy pathway, which is consistent with the findings of Ermak et al. (2012). Previous studies have suggested that MPTP opening is associated with mitophagy (RodriguezEnriquez et al., 2004; 2009). Ermak et al. (2012) also demonstrated that Rcan1-1L caused mPTP opening, which may the reason for Rcan1-1L-activated mitophagy. In the present study, we found that overexpression of Rcan1-1L markedly increased mPTP opening under hypoxic conditions, implying that overexpression of Rcan1-1L stimulates mitophagy through regulating mPTP. However, the mechanism through which Rcan1-1L regulates $\mathrm{mPTP}$ opening remains unknown. It has been reported that Rcan1-1 activates the mitochondrial adenosine transmitter, a component of MPTP, which may explain why Rcan1$1 \mathrm{~L}$ has a functional effect on MPTP (Chang and Min, 2005). However, our findings concerning the impact of Rcan1-1L overexpression-induced mitophagy on cell growth and survival are inconsistent with the findings of Ermak et al. (2012). They found that Rcan1-1L overexpression reduced cell survival under normoxic conditions. In our study, we also demonstrated that overexpression of Rcan1-1L inhibited cell growth under normoxic conditions. However, we found that Rcan1-1L overexpression increased cell survival under hypoxic conditions. Interestingly, our findings were in line with a more recent study which demonstrated that Rcan 1-1L protected cardiomyocytes against hypoxia-induced apoptosis via induction of mitophagy (Yan et al., 2014). Given the role of mitochondria as an energy source for cells, one can speculate that fewer mitochondria not surprisingly impair cell growth and survival. However, damaged mitochondria caused by hypoxic treatment in cardiomyocytes has been reported to contribute to generate excessive ROS that activates cell death leading to myocardial damage (Chatterjee et al., 2002; Nakagawa et al., 2005; Neubauer, 2007). Here, we found that Rcan $1-1 \mathrm{~L}$ overexpression significantly decreased the hypoxia-induced mitochondrial depolarization implying that Rcan1-1L overexpression contributed to maintain the homeostasis of mitochondria under hypoxia. Therefore, their clearance will be beneficial for cell growth and survival under hypoxcic conditions. In lung caner cells, New- 
castle disease virus favors its replication by blocking apoptosis through induction of mitophagy (Meng et al., 2014). The precise biological impact of mitophagy on the cell may be dependent on its current status and extracellular environment. A side effect of mitophagy is a decrease in ATP production. Here, we also demonstrated that overexpression of Rcan1-1L decreased ATP levels. However, cardiomyocyte survival is more important than energy production and demand under hypoxia. Interestingly, it has been suggested that lower cellular ATP levels might be beneficial for cell survival through the induction of cell-cycle arrest (Mandal et al., 2005). Taken as a whole, Rcan1-1Linduced mitophagy is a potential therapeutic approach for ischemia-associated heart diseases.

Recently, mitophagy has been proposed to play a role in the treatment of ischemic heart disease (Jimenez et al., 2014). Selectively removing damaged mitochondria and leaving robust mitochondria is beneficial for cells trying to resist ischemic stress. Inhibition of autophagy flow by knockdown of ATG 5, a critical gene for autophagy flow, resulted in numerous dysfunctional mitochondria and led to rapid myocardial failure in a mouse model (Nakai et al., 2007). Interestingly, knockdown of p53, a common cancer suppressor gene, can protect the myocardium against ischemia through upregulating mitophagy in cardiomyocytes (Hoshino et al., 2012). Recently, Huang et al. (2011) demonstrated that ischemic preconditioning provided cardioprotection though regulating the translocation of Parkin into mitochondria to induce mitophagy. It was found that loss of Parkin leads to an accumulation of damaged mitochondria that contributed to myocardial dysfunction (Kubli et al., 2013). In the present study, we found that Parkin translocated from the cytosol into mitochondria in cells overexpressing Rcan1-1L, indicating that Rcan1-1L regulates mitophagy through Parkin. However, whether Rcan1-1L also regulates mitochondrial degradation through other pathways such as Nix/Bnip3 or Fundc1 requires further investigation.

Considering that selective removal of damaged mitochondria not only reduces ROS production but also inhibits activation of the mitochondria-mediated apoptotic pathway, targeting mitophagy is a potential and promising therapeutic approach for the treatment of ischemic heart disease. In the present study, we have demonstrated that Rcan1-1L overexpression specifically activates mitophagy and increases cell survival under hypoxic conditions, implying that Rcan $1-1 \mathrm{~L}$ should be adopted as a molecular target for gene therapy of ischemic heart disease. It is noteworthy that long-term expression of Rcan1-1L promoted apoptosis (Wu and Song, 2013), which might be due to the induction of excessive mitophagy, resulting in cell death. Therefore, considering the application of mitophagy in therapy, induction of mitophagy must be tightly controlled in order to avoid excessive mitophagy-induced cell death. This huge challenge will have to be overcome before mitophagy can be used to treat ischemia-related diseases.

\section{ACKNOWLEDGMENTS}

This study was supported by grants from the National Natural Science Foundation of China (NO. 81170185) and (NO. 30871041).

\section{REFERENCES}

Campello, S., Strappazzon, F., and Cecconi, F. (2014). Mitochondrial dismissal in mammals, from protein degradation to mitophagy. Biochim. Biophys. Acta 1837, 451-460.

Chan, N.C. Salazar, A.M. Pham, A.H., Sweredoski, M.J., Kolawa, N.J., Graham, R.L., Hess, S., and Chan, D.C. (2011). Broad activation of the ubiquitin-proteasome system by Parkin is critical for mitophagy. Hum. Mol. Genet. 20, 1726-1737.

Chang, K.T., and Min, K.T. (2005). Drosophila melanogaster homolog of Down syndrome critical region 1 is critical for mitochondrial function. Nat. Neurosci. 8, 1577-1585.

Chatterjee, S., Stewart, A.S., Bish, L.T., Jayasankar, V., Kim, E.M., Pirolli, T., Burdick, J., Woo, Y.J., Gardner, T.J., and Sweeney, H.L. (2002). Viral gene transfer of the antiapoptotic factor Bcl-2 protects against chronic postischemic heart failure. Circulation 106, 1212-217.

Cho, D.H., Nakamura, T., and Lipton, S.A. (2010). Mitochondrial dynamics in cell death and neurodegeneration. Cell Mol. Life Sci. 67, 3435-3447.

Cossarizza, A., Ceccarelli, D., and Masini, A. (1996). Functional heterogeneity of an isolated mitochondrial population revealed by cytofluorometric analysis at the single organelle level. Exp. Cell Res. 222, 84-94.

Crawford, D.R., Leahy, K.P., Abramova, N., Lan, L., Wang, Y., and Davies, K.J. (1997). Hamster adapt78 mRNA is a down syndrome critical region homologue that is inducible by oxidative stress. Arch. Biochem. Biophys. 342, 6-12.

Davie,s K.J., Ermak, G., Rothermel, B.A., Pritchard, M., Heitman, J., Ahnn, J., Henrique-Silva, F., Crawford, D., Canaider S., Strippol P., et al. (2007). Renaming the DSCR1/Adapt78 gene family as RCAN: regulators of calcineurin. FASEB J. 21, 3023-3028.

Ding, W.X., and Yin, X.M. (2012). Mitophagy: mechanisms, pathophysiological roles, and analysis. Biol. Chem. 393, 547564

Ermak, G., Harris, C.D., and Davies, K.J. (2002). The DSCR1 (Adapt78) isoform 1 protein calcipressin 1 inhibits calcineurin and protects against acute calcium-mediated stress damage, including transient oxidative stress. FASEB J. 16, 814-824.

Ermak, G., Harris, C.D., Battocchio, D., and Davies, K.J. (2006). RCAN1 (DSCR1 or Adapt78) stimulates expression of GSK3beta. FEBS J. 273, 2100-2109.

Ermak, G., Pritchard, M.A., Dronjak, S., Niu, B., and Davies, K.J. (2011). Do RCAN1 proteins link chronic stress with neurodegeneration? FASEB J. 25, 3306-3311.

Ermak, G., Sojitra, S., Yin, F., Cadenas, E., Cuervo, A.M., and Davies, K.J. (2012). Chronic expression of RCAN1-1L protein induces mitochondrial autophagy and metabolic shift from oxidative phosphorylation to glycolysis in neuronal cells. J. Biol. Chem. 287, 14088-14098.

Fuentes, J.J., Pritchard, M.A., Planas, A.M., Bosch, A., Ferrer, I., and Estivill, X. (1995). A new human gene from the down syndrome critical region encodes a proline-rich protein highly expressed in fetal brain and heart. Hum. Mol. Genet. 4, 19351944.

Fuentes, J.J., Pritchard, M.A., and Estivill, X. (1997). Genomic organization, alternative splicing, and expression patterns of the DSCR1 (Down syndrome candidate region 1) gene. Genomics 44, 358-361.

Goldberg, A.L. (2003). Protein degradation and protection against misfolded or damaged proteins. Nature 426, 895-899.

Hoshino A., Matoba, S., Iwai-Kanai, E., Nakamura, H., Kimata, M., Nakaoka, M., Katamura, M., Okawa, Y., Ariyoshi, M., Mita, Y., et al. (2012). p53-TIGAR axis attenuates mitophagy to exacerbate cardiac damage after ischemia. J. Mol. Cell Cardiol. 52, 175-184

Huang, C., Andres, A.M., Ratliff, E.P., Hernandez, G., Lee, P., and Gottlieb, R.A. (2011). Preconditioning involves selective mitophagy mediated by Parkin and p62/SQSTM1. PLoS One 6, e20975.

Jimenez, R.E., Kubli, D.A., and Gustafsson, A.B. (2014). Autophagy and mitophagy in the myocardium: therapeutic potential and concerns. Br. J. Pharmacol. 171, 1907-1916.

Kim, S.S., Jang, S.A., and Seo, S.R. (2013). CREB-mediated Bcl-2 expression contributes to RCAN1 protection from hydrogen peroxide-induced neuronal death. J. Cell Biochem. 114, 11151123.

Klionsky, D.J., Abdalla, F.C., Abeliovich, H., Abraham, R.T., Acevedo-Arozena, A., Adeli, K., Agholme, L., Agnello, M., Agostinis, P., Aguirre-Ghiso, J.A., et al. (2012). Guidelines for the use and interpretation of assays for monitoring autophagy. Autophagy 8, 445-544

Koopman, W.J., Willems, P.H., and Smeitink, J.A. (2012). Monogenic mitochondrial disorders. N. Engl. J. Med. 366, 1132 1141.

Kubli, D.A., Zhang, X., Lee, Y., Hanna, R.A., Quinsay, M.N., Nguyen, 
C.K., Jimenez, R., Petrosyan, S., Murphy, A.N., and Gustafsson, A.B. (2013). Parkin protein deficiency exacerbates cardiac injury and reduces survival following myocardial infarction. J. Biol. Chem. 288, 915-926.

Lemasters, J.J. (2005). Selective mitochondrial autophagy, or mitophagy, as a targeted defense against oxidative stress, mitochondrial dysfunction, and aging. Rejuvenation Res. 8, 3-5.

Liu, L., Feng, D., Chen, G., Chen, M., Zheng, Q., Song, P., Ma, Q., Zhu, C., Wang, R., Qi, W., et al. (2012). Mitochondrial outermembrane protein FUNDC1 mediates hypoxia-induced mitophagy in mammalian cells. Nat. Cell. Biol. 14, 177-185.

Mandal, S., Guptan, P., Owusu-Ansah, E., and Banerjee, U. (2005). Mitochondrial regulation of cell cycle progression during development as revealed by the tenured mutation in Drosophila. Dev. Cell 9, 843-854

Matsuda, N., Sato, S., Shiba, K., Okatsu, K., Saisho, K., Gautier, C.A., Sou, Y.S., Saiki, S., Kawajiri, S., Sato, F., et al. (2010). PINK1 stabilized by mitochondrial depolarization recruits Parkin to damaged mitochondria and activates latent Parkin for mitophagy. J. Cell Biol. 189, 211-221.

Meng, G., Xia, M., Wang, D., Chen, A., Wang, Y., Wang, H., Yu, D., and Wei, J. (2014). Mitophagy promotes replication of oncolytic Newcastle disease virus by blocking intrinsic apoptosis in lung cancer cells. Oncotarget 5, 6365-6374.

Nakagawa, T., Shimizu, S., Watanabe, T., Yamaguchi, O., Otsu, K., Yamagata, H., Inohara, H., Kubo, T., and Tsujimoto, Y. (2005). Cyclophilin D-dependent mitochondrial permeability transition regulates some necrotic but not apoptotic cell death. Nature 434 652-658.

Nakai, A., Yamaguchi, O., Takeda, T., Higuchi, Y., Hikoso, S. Taniike, M., Omiya, S., Mizote, I., Matsumura, Y., Asahi, M., et al. (2007). The role of autophagy in cardiomyocytes in the basal state and in response to hemodynamic stress. Nat. Med. 13, 619-624.

Nakatogawa, H., Suzuki, K., Kamada, Y., and Ohsumi, Y. (2009). Dynamics and diversity in autophagy mechanisms: lessons from yeast. Nat. Rev. Mol. Cell Biol. 10, 458-467.

Neubauer, S. (2007). The failing heart--an engine out of fuel. $\mathrm{N}$. Engl. J. Med. 356, 1140-1151.

Novak, I., Kirkin, V., McEwan, D.G., Zhang, J., Wild, P., Rozenknop, A., Rogov, V., Lohr, F., Popovic, D., Occhipinti, A., et al. (2010). Nix is a selective autophagy receptor for mitochondrial clearance. EMBO Rep. 11, 45-51.

Nunnari, J., and Suomalainen, A. (2012). Mitochondria: in sickness and in health. Cell 148, 1145-1159.

Petronilli, V., Miotto, G., Canton, M., Brini, M., Colonna, R., Bernardi, P., and Di Lisa, F. (1999). Transient and long-lasting openings of the mitochondrial permeability transition pore can be monitored directly in intact cells by changes in mitochondrial calcein fluorescence. Biophys. J. 76, 725-734.

Qin, L., Zhao, D., Liu, X., Nagy, J.A., Hoang, M.V., Brown, L.F., Dvorak, H.F., and Zeng, H. (2006). Down syndrome candidate region 1 isoform 1 mediates angiogenesis through the calcineurin-NFAT pathway. Mol. Cancer Res. 4, 811-820.

Rodriguez-Enriquez, S., He, L., and Lemasters, J.J. (2004). Role of mitochondrial permeability transition pores in mitochondrial autophagy. Int. J. Biochem. Cell Biol. 36, 2463-2472.

Rodriguez-Enriquez, S., Kai, Y., Maldonado, E., Currin, R.T., and Lemasters, J.J. (2009). Roles of mitophagy and the mitochondrial permeability transition in remodeling of cultured rat hepatocytes. Autophagy 5, 1099-1106.

Scherz-Shouval, R., and Elazar, Z. (2011). Regulation of autophagy by ROS: physiology and pathology. Trends Biochem. Sci. 36, 30-38.

Serrano-Candelas, E., Farre, D., Aranguren-lbanez, A., MartinezHoyer, S., and Perez-Riba, M. (2014). The vertebrate RCAN gene family: novel insights into evolution, structure and regulation. PLoS One 9, e85539.

Song, Y., Xiao, Y., Wang, J.M., and Chen, Q. (2014). The different molecular mechanisms of mitophagy between yeast and mammals. Crit. Rev. Eukaryot. Gene Expr. 24, 29-38.

Sun, X., Wu, Y., Chen, B., Zhang, Z., Zhou, W., Tong, Y., Yuan, J., Xia, K., Gronemeyer, H., Flavell, R.A., et al. (2011). Regulator of calcineurin 1 (RCAN1) facilitates neuronal apoptosis through caspase-3 activation. J. Biol. Chem. 286, 9049-9062.

Wang, Y., De Keulenaer, G.W., Weinberg, E.O., Muangman, S., Gualberto, A., Landschulz, K.T., Turi, T.G., Thompson, J.F., and Lee, R.T. (2002). Direct biomechanical induction of endogenous calcineurin inhibitor down syndrome critical region-1 in cardiac myocytes. Am. J. Physiol. Heart Circ. Physiol. 283, H533-539.

$\mathrm{Wu}$, Y., and Song, W. (2013). Regulation of RCAN1 translation and its role in oxidative stress-induced apoptosis. FASEB J. 27, 208221.

Yan, L., Li, Y., Duan, H., Yang, H., Wu, J., Qian, P., Li, B., and Wang, S. (2014). Regulator of calcineurin 1-1L protects cardiomyocytes against hypoxia-induced apoptosis via mitophagy. J. Cardiovasc. Pharmacol. [Epub ahead of print].

Yang, Z., and Klionsky, D.J. (2010). Eaten alive: a history of macroautophagy. Nat. Cell. Biol. 12, 814-822.

Youle, R.J., and Narendra, D.P. (2011). Mechanisms of mitophagy. Nat. Rev. Mol. Cell. Biol. 12, 9-14

Zhang, J., and Ney, P.A. (2009). Role of BNIP3 and NIX in cell death, autophagy, and mitophagy. Cell Death Differ. 16, 939-946.

Zhao, P., Xiao, X., Kim, A.S., Leite, M.F., Xu, J., Zhu, X., Ren, J., and $\mathrm{Li}, \mathrm{J}$. (2008). c-Jun inhibits thapsigargin-induced ER stress through up-regulation of DSCR1/Adapt78. Exp. Biol. Med. (Maywood) 233, 1289-1300. 